\title{
Stars Near A Giant Black Hole
}

\section{J.-P. Luminet}

Observatoire de Paris-Meudon, France

University of California at Berkeley, USA
Accretion into giant black holes that are nuclei for the formation of galaxies is thought to be the most plausible description of the extraordinarily bright, compact sources of radiation observed at the centres of some active galaxies.

But black holes are not only beautiful theoretical concepts; they are also celestial objects, i.e. they probably exist in the Universe. Stellar black holes are suspected to lie in at least three well observed X-ray binary stars: the celebrated Cygnus $\mathrm{X}-1$, first discovered in 1965 by a rocket-borne detector, the dwarf binary AO 620-00 - perhaps the most convincing case, and LMC X-3 in the Large Magellanic Cloud. These systems harbour compact stars whose total mass, deduced from orbital and spectral characteristics, exceeds $3 M_{0}$ (where $M_{0}$ is the solar mass) - a limit above which, according to general relativity, a cold equilibrium configuration such as a white dwarf or a neutron star would be unstable.

\section{Giant Black Holes as Galactic Nuclei}

On a much larger scale, giant black holes were proposed in the 1950's by theoretical astrophysicists as plausible central powerhouses of active galactic nuclei. But even as long ago as the end of the 18th century, Mitchell and Laplace speculated on the existence of celestial bodies, with radii as large as one astronomical unit, the mean density of which was of the same order as that of the planets. Such massive objects would generate a gravitational field strong enough to trap light rays and particles, so they would be invisible. The critical mass calculated by Mitchell

Dr. Jean-Pierre Luminet is a researcher with the Département d'Astrophysique Relativiste et de Cosmologie, Observatoire de Paris Meudon, F-92195 Meudon Cédex having recently spent one year with the Astronomy Department at the University of California at Berkeley. $\mathrm{He}$ is mainly interested in general relativity, black holes and relativistic cosmology and is the author of a book on black holes titled "Les trous noirs" (Belfond/Sciences, Paris) 1987 with a translation in English (Cambridge University Press) 1990. and Laplace was $10^{7} M_{\odot}$, i.e. of the same order of magnitude as the mass concentrations now inferred from observations of many galactic nuclei.

A typical example of a powerful galactic nucleus is the quasar $3 \mathrm{C} 273$. Its intrinsic luminosity is 1000 times the luminosity of a whole galaxy such as the Milky Way, whereas its radiation is generated in a very compact region (as small as the solar system). Energetic considerations suggest that this tremendous amount of radiation is most likely caused by the release of gravitational potential energy and not by nuclear processes, which are not sufficiently efficient. Gas accretion into a giant black hole is now considered as the most appropriate basic description of the central engine [1].

\section{Supporting evidence}

Paradoxically, the most convincing observations supporting the massive black hole hypothesis come from galactic nuclei which are far from being active, namely the Galactic Centre, the nuclei of the Andromeda Galaxy and of its dwarf elliptic satellite $\mathrm{M} 32$, and other nearby ordinary galaxies. Mass concentrations in these galactic nuclei can be inferred from [2]:

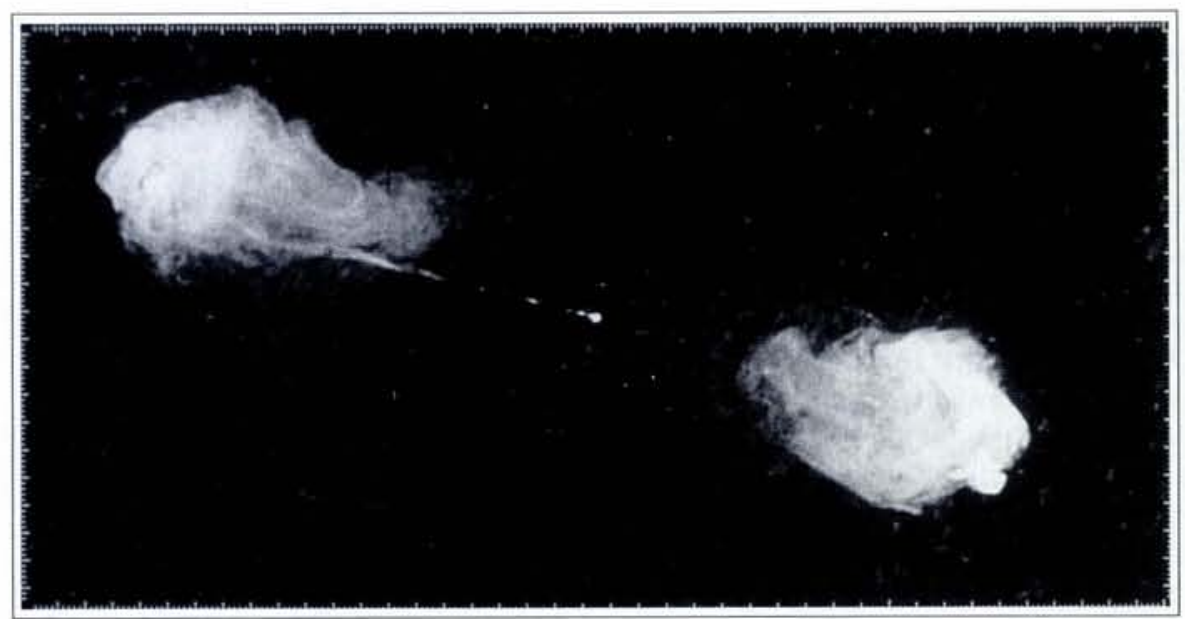

Fig. 2 - The elliptical galaxy Messier 87. Photometry of the central region together with measurements of the stellar velocity dispersion suggest the presence of a central dark mass of several thousand million solar masses. However, the choice between a supermassive black hole and a tight stellar cluster is still open.
(1) The distribution of light near the centre. A spike in the luminosity profile may signal an unusual accumulation of stars around a massive central body.

(2) Dynamic arguments. Spectroscopic measurements give the velocity dispersion of stars or emitting clouds orbiting

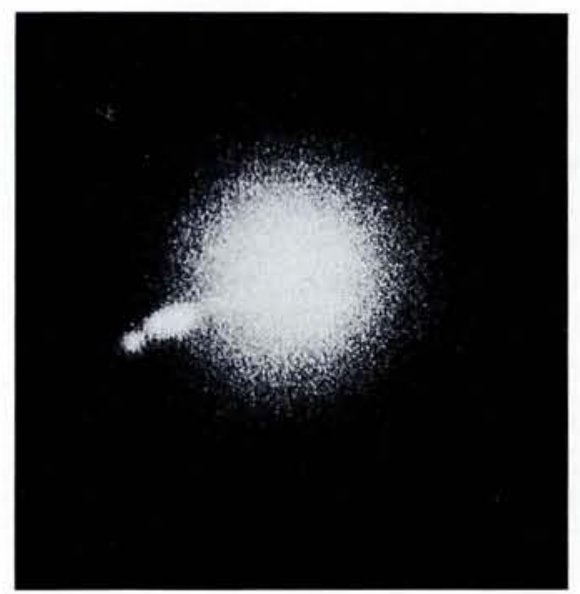

Fig. 1 - The giant radiogalaxy Cygnus A. This powerful radio source exhibits huge jets which may correspond to the expulsion of gas along the symmetry axis of a supermassive rotating black hole surrounded by a thick accretion disk. 
the putative black hole. Assuming that the mass distribution is spherical, the mean stellar rotation is circular, and the velocities are isotropic, the total mass within a radius $r$ can be deduced.

Data for all these nearby galaxies are consistent with models involving a compact source ranging in size from one to fifty million solar masses. The repeated occurrence of such features suggests that the presence of massive black holes at the centres of spiral and elliptical galaxies might be quite common (even the rule), and that these huge objects were the seeds for galaxy formation.

\section{Feeding Active Giants}

Now let us focus our attention on a subclass of active galactic nuclei (approximately $1 \%$ of the total) which includes Seyfert galaxies, blazars, radiogalaxies and quasars (Figs. 1, 2). Their luminosities typically lie in the range $10^{44}-10^{48} \mathrm{erg} / \mathrm{sec}$ (the luminosity of the Sun is $10^{33} \mathrm{erg} / \mathrm{sec}$ ). Powering such a bright source by a massive accreting black hole requires the digestion of gas at a rate between $0.01 M_{\odot}$ and $100 M_{\odot}$ per year. Three modes of fuelling are currently invoked by theoreticians:

\section{Accretion of exogenous matter}

A correlation - that is not yet firmly established - appears to exist between the activity of a galactic nucleus and the interaction of its host galaxy with a nearby galactic companion. However, the process of channelling the gas that is tidally stripped from the companion in the direction of the central black hole of the main galaxy is far from understood.

\section{Accretion of galactic gas}

The total amount of gas produced by mass loss during ordinary stellar evolution in an entire spiral galaxy such as the Milky Way is about $1 M_{\odot} / y r$. This could be just large enough to power moderately active galaxies. However, it is hard to believe that all the gas would be focussed towards the centre of the galaxy (for instance, supernovae winds have velocities greater than the escape velocity and would be lost into space). Moreover, elliptic galaxies also show signs of accretion activity in spite of their having no interstellar gas. This type of accretion process could nevertheless be efficient for barred spiral galaxies (see Europhys. News 21 (1990)3) since recent numerical simulations of galactic dynamics suggest that a cascade of bars forming at smaller and smaller scales could bring matter closer and closer to the centre.

\section{Interaction with a surrounding stellar cluster}

The most widely accepted model for central activity in galaxies involves a giant black hole imbedded in a large local cloud of stars. The scattering of stars' orbits within the cloud allows some stars to fall deeply into the central pit and penetrate the so-called accretion radius where the black hole's gravity dominates stellar dynamics. Satisfactory modelling of active galactic nuclei thus requires theoretical understanding of the behaviour of matter moving in a strong gravitational field, and of mechanisms that should be able to extract gas from stars more efficiently than ordinary mass loss due to stellar evolution.

Inside the accretion radius, various processes are likely to release large amounts of gas, energy and radiation. They are:

(a) Ablation of stellar atmospheres by the external radiation field. This process resembles the evaporation of comets on approaching the Sun. However, for these "cometary stars", the outward flux of gas comes from their interaction with a pre-existing accretion structure around a massive black hole. The ablation of stellar atmospheres can therefore only provide a modest fraction of the total fuelling rate.

(b) Disruption of stars by high velocity collisions. When the elative collision velocity between two stars exceeds the stellar escape velocity (about $500 \mathrm{~km} / \mathrm{s}$ for a typical main-sequence star) mass is lost instead of merged. "Disruptive" collisions of this type may be effective inside the so-called collision radius around the black hole - the distance within which the free-fall velocity becomes higher than the stellar escape velocity. This distance increases linearly with the mass of the black hole and is of the order of $10^{13} \mathrm{~km}$ for a $10^{8} M_{\odot}$ black hole interacting with a solar-type star.

(c) Disruption of stars by a tidal field. A black hole generates strong variations in its gravitational field, and any star approaching within a characteristic distance given by the tidal radius will be disrupted. The process of disruption can be grossly understood in the following way: when the orbital timescale of the free-falling star (i.e. the characteristic variation with time of the external gravitational field) becomes shorter than the star's internal dynamic timescale, the star can no longer readjust its configuration to the external perturbation. It enters a regime of growing oscillations that ultimately lead to its disruption. The phenomenon occurs precisely at the point the star penetrates inside the tidal radius, which grows with the mass of the black hole as $M^{1 / 3}$. For a solar-type star and a $10^{8} \mathrm{M}_{\odot}$ black hole, the tidal radius is about $6 \times 10^{8} \mathrm{~km}$.

The gravitational radius of a black hole increases linearly with the mass and must exceed the tidal radius at a sufficiently large mass - the so-called Hills limit, equal to $3 \times 10^{8} M_{\odot}$ for solartype stars. Above this limit, energy release by the tidal disruption of stars becomes inefficient since release occurs inside the black hole from which nothing can escape.

Global energy arguments suggest that the most powerful active galaxies such as quasars require a supermassive black hole $\left(>10^{9} M_{\odot}\right)$ so their main mode of fuelling is likely to involve the collisional disruption of stars.

\section{Stellar Pancakes}

Progress has recently been made in understanding the tidal disintegration process [3]. B. Carter and I working at the Observatoire de Meudon made the first prediction that the external tidal field generated by a large black hole detonates an explosive nucleosynthesis in a star plunging deep inside the tidal radius. We proved that in the most extreme form of tidal disruption, the stellar core, instead of being continuously decompressed and broken into filamentary clouds, first undergoes a transitory phase of huge compressional flattening and heating, whose effects on the dynamics and the chemical composition of the released gas may be of a primary importance.

The phenomenon can be understood by recalling that inside the tidal radius, the external gravitational forces rapidly begin to dominate the internal pressure and self-gravitational forces inside the star, so the star's particles initially undergo free-fall motion. The particles then tend to be focused towards the orbital plane as the star approaches the periastron, and overall compression occurs. Of course, as the volume of the star moves towards zero, the internal pressure suddenly reacts and the star bounces abruptly to an expansion phase accompanied by the ejection of gas. The star must consequently pass through a fixed point near the periastron of its orbit where it looks like a squeezed tube of toothpaste (Fig. 3). This squeezing effect can be viewed as yielding a pancake in that owing to the high orbital velocity compared to the velocity of sound, it can be considered as taking place simultaneously over all parts of the star. 
The extent of flattening depends critically on the so-called penetration factor, defined as the ratio of the tidal radius to the periastron distance of the orbit (the distance at the point of closest approach). As an example, a solar-type star plunging by a factor 15 inside the tidal radius of a $10^{5} M_{\odot}$ black hole (i.e. along a parabolic orbit with a periastron distance $9.1 \times 10^{5} \mathrm{~km}$ ) is compressed by a factor of $\approx 750$ and heated by a factor of $\approx 80$ during $\approx 0.1$ second.

An additional, interesting and purely relativistic effect of the Schwarzschild gravitational field for a non-rotating black hole is that, when the plunging orbit has a double point located inside the tidal radius, the star passes through several squeezing points and suffers successive pancake flattenings separated by a few seconds (Fig. 4).

\section{Nucleosynthesis}

Recent numerical simulations of the flattening process [4] examined mainsequence stars orbiting $10^{5}-10^{6} M_{\odot}$ black holes, and degenerate stars (white dwarfs, helium stars) grazing $10^{3} M_{\odot}$ black holes. The equation of state of stellar material was treated as a mixture of a non-relativistic perfectly ionic gas, a semi-degenerate semi-relativistic electron gas, and a photon gas. Typically, the central temperature increased by a factor of $\approx 50$ and the density by a factor of $\approx 100$ within a few milliseconds with a penetration factor of 15 for a main-sequence star, and of only 5 for a degenerate dwarf.

The dynamics of the star was then coupled with a network of nuclear reactions. In the prevailing thermodynamic conditions, neutron producing reactions are negligible and the main nuclear flow is dominated by helium capture or proton capture reactions. Calculations of the nuclear power output and changes in chemical composition confirmed that, for a sufficiently high penetration factor, tidal squeezing may trigger explosive nucleosynthesis in the stellar core and release more nuclear energy than the stellar gravitational binding energy (up to more than $10^{49}$ ergs). The principal isotopes that are produced are ${ }^{15} \mathrm{~N},{ }^{25} \mathrm{Mg},{ }^{26} \mathrm{Mg},{ }^{26} \mathrm{Al}$ and ${ }^{27} \mathrm{Al}$ in main-sequence stars, and ${ }^{28} \mathrm{Si}$ in helium degenerate stars. In main-sequence stars, about half of the stellar debris escapes the black hole in the form of a high-velocity hot wind capable of blowing away the intervening gas clouds. The other half falls quickly into the hole, producing a sudden burst of luminosity. In broken-up degenerate stars, the gas remains bound to the hole.

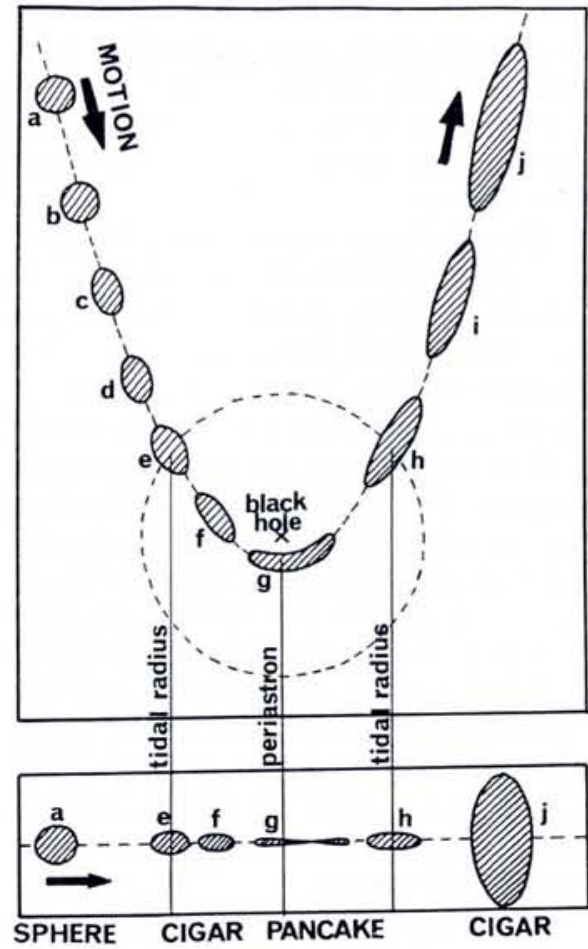

Fig. 3 - Deformations of a star penetrating deeply within the tidal radius of a black hole (for the sake of clarity, the size of the star has been enlarged compared to the orbital distance scale). Upper: configurations as projected in the orbital plane; lower: configurations in the direction orthogonal to the orbital plane. Bouncing occurs in the vicinity of the periastron to give a squeezed star that looks like a pancake at the high orbital velocity.

Fig. 4 - Tidal squeezing of a star in the relativistic gravitational field of a non-rotating black hole. Upper: trajectory of the centre of mass. The position of the black hole and its Schwarzschild radius are indicated at the origin of coordinates; the circle represents the tidal radius. Lower: principle axes $\left(a_{1}, a_{2}, a_{3}\right)$ of the star. The orbital timescale is in seconds and the dashed vertical lines indicate the times at which the star passes inside and out of the tidal radius.

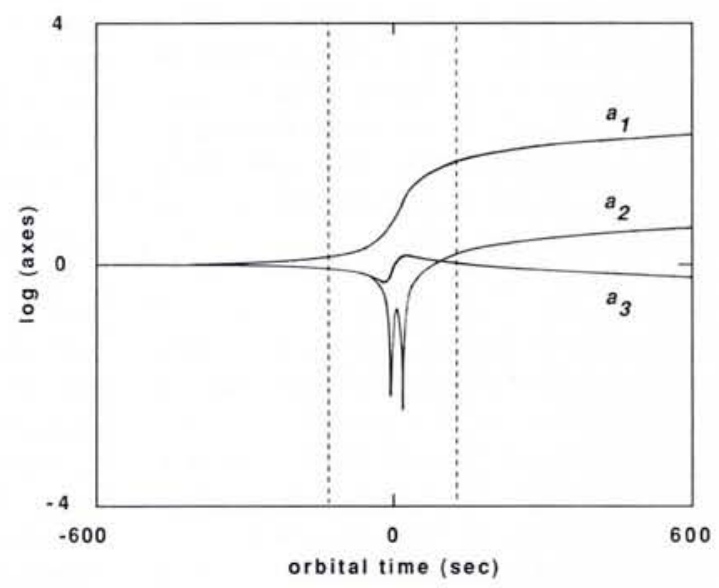

In many respects the pancake stars can be considered as supernovae explosions occurring "by accident", owing to a strong gravitational field that basically acts as the detonator for the thermonuclear explosion. The external gravitational field generated by the neighbouring black hole compresses the star "from the outside" and triggers the detonation. On the other hand, in supernovae the self-gravitational field of the star mines "from the inside" the stellar stability, and the resulting collapse of the stellar core triggers the explosion. In both cases there is a release of nuclear energy and the ejection of gas.

Like supernovae, stellar pancakes are sites of nucleosynthesis that are able to build up heavy elements and enrich the interstellar medium. The calculations show however, that the nature and the relative abundances of the isotopes produced in stellar pancakes differ from those of isotopes produced in supernovae (their initial composition is not the same). With improved resolution in spectroscopy in the near future, it should be feasible to detect the characteristic isotopes arising in gas clouds orbiting the centres of active galaxies. Such an identification would provide an additional observational signature of the existence of giant black holes operating as the central engines of activity.

With the availability of more powerful computers, several groups are carrying 
out full three-dimensional hydrodynamic calculations to check our results more accurately. Hydrodynamic simulations of fuelling processes involving stellar collisions have also been performed recently, and the corresponding mass losses were calculated [5].

\section{Disruption Frequency}

Astrophysical interest in explosive stellar disruption by massive black holes (via tidal forces or via collisions) is constrained however by the statistical frequency of such events. Collisional type disintegration depends sensitively on the stellar distribution of stars around the black hole. It is clear that the presence of a massive central object in a stellar cluster modifies the stellar distribution, and this is likely to be one of the reasons why globular clusters (which probably harbour tight binaries instead of massive black holes) and stellar clusters in galactic nuclei have different stellar distributions.

The stellar distribution function depends, in general, on the position, star velocity and time. Assuming spherical symmetry, the dependance can be reduced to two parameters: the orbital energy and the angular momentum per unit mass of a star. Another simple assumption, that the stellar distribution is independent of time, allows some analytical work to be done. However, stars with bound and unbound orbits provide different contributions to the stellar number density.

\section{Bound and unbound orbits}

Stars on unbound orbits have a Maxwellian distribution of velocities outside the accretion radius (the radius of gravitational influence of the black hole) and their distribution is given to a good approximation by the celebrated isothermal sphere, characterized by a mass $M_{\mathrm{c}}$ and a core density $n_{\mathrm{c}}$. For a typical galactic nucleus, $M_{\mathrm{c}} \approx 10^{6}$ to $3 \times 10^{8}$ $M_{\odot}$ and $n_{c} \approx 3 \times 10^{-36}-3 \times 10^{-34} \mathrm{~km}^{-3}$ (the corresponding dispersion velocity being $\approx 150-1000 \mathrm{~km} / \mathrm{s}$ ).

Unbound stars have a number density $n(r) \sim r^{-1 / 2}$ inside the accretion radius so it is possible to calculate their rate of penetration into the tidal radius and the collision radius with a view to estimating the total mass of gas released by stellar disruptions. The tidal contribution turns out to be $\approx 1 M_{\odot} / y r \times M_{8}^{4 / 3}$ and the collisional contribution $\approx 0.1$ $M_{\odot} / y r \times M_{8}^{3}$ (where $M_{8}$ is the mass of the black hole in units of $10^{8} M_{\odot}$ ).

Bound orbits are confined within the accretion radius. The velocity distribution is no longer isotropic because the stars disappear as soon as they pene- trate the tidal radius. The stellar number density reflects an accumulation of stars in the vicinity of the collision radius - a "cusp" in the stellar distribution that favours mostly collisional disruptions.

More general numerical simulations - taking into account the anisotropy of stellar velocities and time dependance - have been performed [6]. The growth of the central black hole is governed by the capture of the stellar debris: after a brief phase of maximum activity, the stellar cluster is exhausted and the black hole accretion rate as well as the accretion luminosity decrease with time as $t^{-1.5}$. The numerical results confirm the idea that moderately active galaxies can be powered by tidal disruption of stars around a $10^{8} M_{\odot}$ black hole, whereas the more powerful quasars require stellar collisions around a $10^{9} M_{\odot}$ black hole, or accretion of extragalactic matter.

The modelling of active galaxies is still in its infancy, and much theoretical work (guided by observations) is needed. Some important issues have not yet been taken into account, such as effects arising from encounters of stars within the accretion disk and the influence of multiple massive black holes. Indeed, some giant galaxies located at the cores of clusters of galaxies indeed show evidence for a multiplicity of active centres.

\section{Conclusions}

The study of the interactions between stars and a giant black hole is extremely interdisciplinary, involving general relativity, stellar structure, nucleosynthesis and galactic dynamics. The production of specific heavy isotopes in stellar pancakes, as well as dynamic features of ejected gases, should be detected shortly using advanced spectroscopic techniques. They may constitute definite signatures of the existence of large black holes in the cores of galaxies.

\section{REFERENCES}

[1] Frank J., King A.R. and Raine D., Accretion Power in Astrophysics (Cambridge University Press) 1985.

[2] Kafatos M., Ed., Supermassive Black Holes (Cambridge University Press) 1988. [3] Carter B. and Luminet J.-P., Nature 296 (1982) 211; Luminet J.-P., Ann. Phys. Fr. 10 (1985) 101; Luminet J.-P., Gravitation in Astrophysics, Eds. B. Carter and J. Hartle (Plenum Press) 1987.

[4] Luminet J.-P. and Pichon B., Astron. Astrophys. 209 (1989) 85.

[5] Benz W. and Hills J.G., Astrophys. J. 326 (1987) 614.

[6] Duncan M. and Shapiro S., Astrophys. J. 268 (1983) 56.

\section{Spanish Spending}

To redress the woeful state of science and technology in Spain, the newly elected national government decided in 1982 to increase government spending on R. and D. from $0.35 \%$ of GNP in 1983 to a planned $1.3 \%$ by 1992 (which is still roughly half that for France and the Federal Republic of Germany). A three-year National Plan was launched in 1988 and its Secretary General recently reported that spending in 1989 had reached $0.9 \%$ of gross national product, divided among 19 principal themes and five special programmes.

The Plan comprises market oriented $\mathrm{Na}$ tional Programmes of the Permanent Interministerial Commission of Science and Technology (CICYT), the Sectorial Programmes for basic research of the Ministry for Science and Education, two horizontal programmes (high energy physics and technology transfer), and special nationallyfunded programmes that can be set up by any of the Spain's 17 autonomous communities. Catalonia, for example, with 25 per cent of Spain's GNP has a major programme in fine chemicals.

\section{New Materials and Universities Gain}

Of the Plan's 19703 million pesetas spending in 1989 about $25 \%$ went to form researchers, resulting in a threefold increase in the number of university scholarships since 1983. The remainder covered joint projects with industry $(21 \%)$ and infrastructure investments and project financing $(54 \%)$ where the new materials theme was the winner (with $16.2 \%$ ) ahead of information technologies and biotechnology (with 14.5 and $13.7 \%$ respectively). The largest autonomous programme (fine chemicals) represented $1.3 \%$ and high energy physics roughly $3.8 \%$.

The universities have benefited most so far from the increase in research spending. They have been awarded $56 \%$ of the infrastructure investments as compared with $22 \%$ for the CSIC which is the national research organization grouping 2000 researchers in numerous centres. The intention is to strengthen the universities' research capacity and to direct it away from the traditional bias for fundamental research towards applied sciences that require greater spending on experimental facilities.

\section{Links with EC Programmes}

It was planned right from the start to couple the National Plan with European Community programmes. For instance, proposals for research projects (in both the sectorial and national programmes) can be submitted anytime if they form part of an EC proposal; otherwise there are deadlines. The policy has worked since from the time it joined the EC until the end of 1988, Spain had received $141 \mathrm{MECU}$ for $R$. and $D$. from the Community (almost exactly the National Plan's 1989 budget). 